\title{
Scalable Production of Genetically Engineered Nanofibrous Macroscopic Materials via Filtration
}

\section{Citation}

Courchesne, Dorval, Noémie-Manuelle, Anna Duraj-Thatte, Pei Kun R. Tay, Peter Q. Nguyen, and Neel S. Joshi. 2016. "Scalable Production of Genetically Engineered Nanofibrous Macroscopic Materials via Filtration." ACS Biomaterials Science \& Engineering (October 26). doi:10.1021/ acsbiomaterials.6b00437.

\section{Published Version}

10.1021/acsbiomaterials.6b00437

\section{Permanent link}

http://nrs.harvard.edu/urn-3:HUL.InstRepos:41017809

\section{Terms of Use}

This article was downloaded from Harvard University's DASH repository, and is made available under the terms and conditions applicable to Open Access Policy Articles, as set forth at http:// nrs.harvard.edu/urn-3:HUL.InstRepos:dash.current.terms-of-use\#OAP

\section{Share Your Story}

The Harvard community has made this article openly available.

Please share how this access benefits you. Submit a story.

\section{Accessibility}




\section{Scalable Production of Genetically Engineered}

\section{Nanofibrous Macroscopic Materials via Filtration}

Noémie-Manuelle Dorval Courchesne ${ }^{\S \dagger}$, Anna Duraj-Thatte ${ }^{\S \neq ⿱ 亠 巾}$, Pei Kun R. Tay ${ }^{\dagger \neq}$, Peter Q.

Nguyen ${ }^{\dagger}$, Neel S. Joshi ${ }^{*}$

${ }^{\S}$ These authors contributed equally.

${ }^{\dagger}$ Wyss Institute for Biologically Inspired Engineering, Harvard University, Boston, MA, United States; ${ }^{*}$ School of Engineering and Applied Sciences, Harvard University, Cambridge, MA, United States

Email : neel.joshi@wyss.harvard.edu, Phone : 617-432-7730

KEYWORDS. Curli fibers, Amyloids, Filtration, Protein-based materials, Thin films

ABSTRACT.

As interest in using proteins to assemble functional, biocompatible and environmentallyfriendly materials is growing, developing scalable protocols for producing recombinant proteins with customized functions coupled to straightforward fabrication processes is becoming crucial. Here, we use E. coli bacteria to produce amyloid protein nanofibers that are key constituents of the biofilm extracellular matrix, and show that protein nanofiber aggregates can be purified using a fast and easily accessible vacuum filtration procedure. With their extreme resistance to heat, 
detergents, solvents and denaturing agents, engineered curli nanofibers remain functional throughout the rigorous processing, and can be used to assemble macroscopic materials directly from broth culture. As a demonstration, we show that engineered curli nanofibers can be fabricated into self-standing films while maintaining the functionality of various fused domains that confer new specific binding activity to the material. We also demonstrate that purified curli fibers can be disassembled, reassembled into thin films, and recycled for further materials processing. Our scalable approach, which combines established purification techniques for amyloid fibers, is applicable to a new class of recombinant amyloid proteins whose sequence can be easily tailored for diverse applications through genetic engineering.

\section{INTRODUCTION.}

The unique self-assembling properties of amyloid proteins make them attractive nanofibrous materials for a variety of applications. They readily form high surface area networks with nanoscale pore sizes, are highly resistant to harsh environments, and can be used under various stages of assembly (monomeric proteins, oligomers, fiber or large aggregates). ${ }^{1}$ Furthermore, their proteinaceous structure lends itself to structural customization, whereby the amyloid fibers serve as a scaffold for the display of protein and peptide domains with various functions. ${ }^{2}{ }^{3}{ }^{4}$ Recently, attempts at controlling the morphology, size and shape of amyloid proteins were achieved by varying assembly conditions, ${ }^{567}$ and they are beginning to be used for various applications including, water purification, ${ }^{8}$ tissue engineering scaffolds, drug delivery, 
templates for polymerization, ${ }^{9}$ self-assembling catalysts for nanomaterial assembly or components of composite films, among others. ${ }^{1}{ }^{10}$

Most reports of amyloid-based materials use either short synthetic peptides or proteins isolated from natural sources as starting components. Naturally occurring proteins are attractive because they can be abundant and readily available. Indeed, many proteins that have no known amyloidogenicity in their natural environment can easily be induced to self-assemble into amyloids by various denaturing treatments. ${ }^{11} 12$ However, in addition to easily inducing amyloid formation, it would also be advantageous to have control over the sequence of the selfassembling unit in order to tailor the material for specific applications. One approach to this is to use solid phase techniques to chemically synthesize the desired sequences. While this works for short ( $<40$ amino acid) sequences, it comes at the expense of scalability and lacks the selfpropagating ability of biological entities for the production of living materials. Alternatively, larger amyloidogenic proteins with customized sequences can be produced recombinantly. ${ }^{10}$ In fact, the ability to tune the sequences of amyloid proteins can lead to enhancements of their selfassembling properties, increased stability, better processability, and easily accessible modifications with functional pendant domains. ${ }^{13}{ }^{14}$ We are particularly interested in curli nanofibers, a class of functional amyloid that is naturally produced by Escherichia coli for the purposes of surface adhesion and biofilm formation. ${ }^{15}{ }^{16}$ Given the ease with which E. coli can be engineered to produce recombinant proteins, these curli nanofibers are of great interest for producing genetically engineered materials. In fact, engineered curli fibers displaying fused protein and peptide domains have been used to imbue bacterial biofilms with a variety of functions, including specific binding to metallic surfaces, antibody and enzyme display, and the ability to template inorganic nanoparticle growth. ${ }^{17} 3^{18} 1920$ This makes curli-based materials 
potentially useful for applications in custom fabricated surface coatings, biocatalysts, and electronically conductive materials. We have developed one such system, which we call BiofilmIntegrated Nanofiber Display (BIND). ${ }^{4}$

Although BIND exhibits much promise as a platform for the manufacture of biologically fabricated materials, scalability remains an unaddressed challenge, especially for recombinantly expressed proteins. Most purification protocols for curli fibers were developed to study their biological and self-assembly properties, and consequently are not optimized to achieve high yields. Accordingly, some purification routes use conventional approaches, including affinity chromatography, centrifugation, preparatory SDS-PAGE, or salt precipitation. ${ }^{15} 2122$ Yields of purified curli fibers are rarely reported for such techniques, but are likely in the low milligram or sub-milligram range. Additionally, the procedures require expensive reagents or instrumentation. Apart from the small quantities of materials obtained from these labor-intensive processes, the requirement for an affinity tag can also interfere with some end-goal applications. In order to produce enough curli nanofibers to construct films, gels or other functional materials, a tag-less, simpler, faster and higher yielding method is needed.

Filtration is a common approach to isolating amyloids, and relies on their resistance to denaturation in the presence of detergents, as compared to other proteins in the cell lysate. ${ }^{23}$ As such, it has been applied in the isolation of recombinant amyloid proteins for analysis, ${ }^{24} 25$ and also in the direct fabrication of several composite materials based on naturally derived $\beta$ lactoglobulin. ${ }^{26} 27282930$ Here, we report a streamlined protocol for the purification of curli protein nanofibers that will be of interest to researchers exploring the fundamental biology of these biofilm matrix components, and also at a scale beyond that which has been previously 
published - enough to facilitate their use in materials applications. In contrast to previously reported examples, our method starts with bacterial cultures and uses only filtration and washes with readily available reagents to yield either purified amyloid protein or functionalized filter membrane surfaces. Notably, no centrifugation or cell lysis is required prior to filtration. This approach is made possible by the stability of the assembled CsgA protein in the face of extreme denaturing conditions that are sufficient to lyse bacterial cells and minimize non-specific binding of other proteins to the amyloids. This method exhibit several desirable features: 1) it is simple and fast, 2) it does not require protein tags and does not rely on any binding affinity, 3) it is easily scalable, allowing for the purification of curli fibers from several liters of bacterial cultures, and 4) it is highly versatile and could be used to purify a wide range of genetically engineered amyloid fibers displaying diverse functional groups. Upon optimization of the process, and confirmation of the purity of filtered protein nanofibers, we demonstrate that we can isolate 10s-100s of milligrams per liter of semi-pure amyloid fibers in 30 mins from induced bacterial culture.

Furthermore, we demonstrate the use of this technique to form free-standing thin films composed exclusively of engineered curli amyloid fibers directly from broth culture, all while maintaining the functionality of peptide and protein domains fused to the fibers. We also show that, through the disassembly of fibers purified by filtration, it is possible to assemble thin films on various substrates, and to recycle curli-based materials through disassembly and reassembly cycles. Thus, our protocol represents a scalable adaptation of filter-based approaches to amyloid isolation for two purposes: 1) to purify recombinant amyloid fibers derived from the curli subunit, $\operatorname{Csg} \mathrm{A}$, and 2) to create functional materials directly from bacterial culture using only filtration washing, without any other purification steps. 
MATERIALS AND METHODS.

Cell strains and plasmids: The divergent curli operon regions consisting of $\operatorname{csg} B A C$ and csgEFG were PCR isolated from E. coli K12 substr. W3110 and cloned by overlap extension

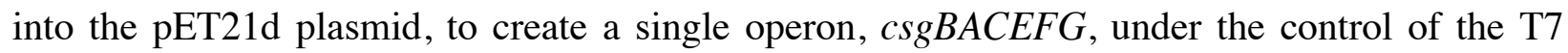
promoter. The $\operatorname{csg} B$ gene was then deleted from the curli operon in order to allow for secretion of the curli fibers directly into the culture medium, free from any anchoring to the bacterial surface. Genes encoding for different CsgA fusions were cloned, with modifications added to the Cterminus: 1) a six-histidine tag (HisTag) was added to allow for immunodetection, 2) a SpyTag sequence and 3) a SpyCatcher sequence (See Table S1 for sequences). The SpyTag peptide and SpyCatcher domain form two halves of an engineered split protein system, driving spontaneous covalent bond formation between any two proteins that are fused to them. ${ }^{31}$ A control plasmid was constructed by cloning the malE gene encoding for the maltose binding protein (MBP) from the W3110 genome into pET21d at the ndeI and bamHI cloning sites. Protein expression was performed in a curli operon deletion mutant, PQN4, an E. coli strain derived from LSR10 $\left(\mathrm{MC} 4100, \Delta \operatorname{csg} A, \lambda(\mathrm{DE} 3), \mathrm{Cam}^{\mathrm{R}}\right)$. PQN4 was constructed by genomic insertion of T7 RNA Polymerase using the $\lambda$ DE3 Lysogenization Kit (Merck-Millipore) into LSR10, verifying for T7dependent pET expression, and then using Lambda Red recombination to knockout the curli operon using pKD46 and pKD3 (plasmids obtained from Coli Genetic Stock Center, Yale University). The deletion of the curli operon was confirmed by sequencing of the curli operon region. Venus yellow fluorescent protein constructs (Venus-SpyTag, Venus-SpyCatcher) were cloned into and expressed from a pDEST14 backbone (Addgene \#35044), followed by purification using a Ni-NTA affinity column. 
Curli nanofiber expression: Transformed PQN4 cells were streaked onto lysogeny broth (LB) agar plates containing $100 \mu \mathrm{g} / \mathrm{mL}$ carbenicillin and $0.5 \%(\mathrm{~m} / \mathrm{v})$ glucose (for catabolite repression of T7RNAP). Colonies were picked from the plates and $5 \mathrm{~mL}$ cultures were inoculated (in LB containing and $100 \mu \mathrm{g} / \mathrm{mL}$ carbenicillin and $2 \%(\mathrm{~m} / \mathrm{v})$ glucose). Cultures were grown overnight at $37^{\circ} \mathrm{C}$. The overnight cultures were diluted 100 -fold in fresh $\mathrm{LB}$ medium with $100 \mu \mathrm{g} / \mathrm{mL}$ carbenicillin and $2 \%(\mathrm{~m} / \mathrm{v})$ glucose, and cultured at $37^{\circ} \mathrm{C}$ until they reached an optical density (OD) at $600 \mathrm{~nm}$ of 0.6 to 0.8 . Cells were pelleted at $4000 \mathrm{x} \mathrm{g}$, and gently resuspended in an induction medium (LB without glucose, containing $0.4 \mathrm{mM} \mathrm{IPTG}$ and $100 \mu \mathrm{g} / \mathrm{mL}$ carbenicillin). Protein expression was allowed to occur at $37^{\circ} \mathrm{C}$ overnight.

Filtration purification of curli nanofibers: After the induction period, guanidinium chloride $(\mathrm{GdmCl})$ was added to the cultures to reach a final concentration of $0.8 \mathrm{M}$ and they were incubated for 1 to 2 hours at $4^{\circ} \mathrm{C}$ prior to filtration. 30 to $50 \mathrm{~mL}$ of the $\mathrm{Gdm}$-containing cultures were then vacuum-filtered onto $47 \mathrm{~mm}$ polycarbonate filter membranes with $10 \mu \mathrm{m}$ pores (EMD Millipore). The filtered biomass was incubated with $5 \mathrm{~mL}$ of $8 \mathrm{M} \mathrm{GdmCl}$ for $5 \mathrm{~min}$, followed by vacuum filtration of the liquid and 3 rinses with $5 \mathrm{~mL}$ of DI water. Next, the filtered biomass was subjected to $5 \mathrm{~mL}$ of an aqueous solution $\left(2 \mu \mathrm{M} \mathrm{MgCl}_{2}\right.$ ) of nuclease (Benzonase ${ }^{\circledR}$, SigmaAldrich, $1.5 \mathrm{U} / \mathrm{mL}$ ) for $10 \mathrm{~min}$, followed by vacuum filtration to remove the liquid and 3 rinses with $5 \mathrm{~mL}$ of DI water. Finally, $5 \mathrm{~mL}$ of $5 \%(\mathrm{~m} / \mathrm{v})$ sodium dodecyl sulfate (SDS) in water was incubated on the filter for $5 \mathrm{~min}$, followed by vacuum filtration of the liquid and 5 rinses with 5 $\mathrm{mL}$ of DI water. Semi-purified curli nanofibers were removed from the filter membrane by gently scraping the filter with a flat spatula. Purified curli nanofibers were lyophilized and subsequently stored at $4^{\circ} \mathrm{C}$. 
Assessment of curli expression and deposition on filters: Curli nanofiber expression was routinely confirmed directly from suspension culture using a Congo Red pull down assay, as described previously. ${ }^{4}$ Briefly, $1 \mathrm{~mL}$ of culture expressing curli was centrifuged and the pellet was resuspended in phosphate buffer. Congo Red was added to a concentration of $0.00015 \%$ $(\mathrm{m} / \mathrm{v})$. After a 10 minute incubation, cells were centrifuged and the absorption at $490 \mathrm{~nm}$ of the supernatant was measured to quantify the amount of Congo Red that did not bind to the cells. The deposition of curli nanofibers on filter membranes was also assessed using Congo Red dye binding. After all the filtration steps, $5 \mathrm{~mL}$ of a $0.015 \%(\mathrm{~m} / \mathrm{v})$ of Congo Red dye was incubated on the filters for $10 \mathrm{~min}$, followed by vacuum filtration of the liquid and 3 rinses with $5 \mathrm{~mL}$ of DI water. As controls, Congo Red dye was also incubated on clean filter membranes and on membranes with control cells expressing MBP instead of curli fibers.

Fabrication of free-standing curli films: Purified curli nanofibers on polycarbonate filter membranes were crosslinked by immersing the membrane in a $2 \%(\mathrm{~m} / \mathrm{v})$ glutaraldehyde and 2 $\%(\mathrm{~m} / \mathrm{v})$ paraformaldehyde solution for 2 hours at room temperature. After water rinses, the polycarbonate filter membranes with crosslinked fibers were placed on a Teflon support membrane, and immersed in a dichloromethane bath for approximately 1 minute to dissolve the polycarbonate filter membrane. Free-floating curli nanofiber films were collected from the dichloromethane bath using the Teflon support and allowed to dry in air for an hour. Dried curli films were gently peeled off the Teflon support.

Assessing the activity of CsgA-SpyTag and CsgA-SpyCatcher fusions on filter membranes and free-standing curli films: Venus yellow fluorescent protein fusions ${ }^{32}$ containing either SpyTag or SpyCatcher were used to assess the binding activity of engineered curli nanofibers, as described previously. ${ }^{4}$ Venus-SpyCatcher and Venus-SpyTag fusion proteins were produced 
recombinantly. The Venus fusion protein solutions were diluted to $10 \mu \mathrm{M}$ in $50 \mathrm{mM}$ phosphate buffer pH 7.2 and incubated on filter membranes with purified CsgA-SpyTag or CsgASpyCatcher nanofibers for 90 minuteat $4^{\circ} \mathrm{C}$. The liquid was then filtered through, and the membrane was rinsed with 5 times with $5 \mathrm{~mL}$ of DI water. To exclude non-specific binding on filter membranes, the following controls were also incubated with Venus-SpyCatcher or VenusSpyTag: 1) purified CsgA-HisTag nanofibers, 2) cells expressing only MBP and subjected to the same filtration treatment, and 3) bare untreated filter membranes. The same protocol was used for Venus-SpyCatcher binding on free-standing CsgA-SpyTag curli films.

Assembling of thin films with dissolved curli fibers: Curli fibers purified via filtration were dissolved in a 1:1 hexafluoroisopropnaol (HFIP):trifluoroacetic acid (TFA) solution, with sonication for $1 \mathrm{~h}$. After complete dissolution, the fibers were reformed by dropcasting the CsgA containing solutions directly onto oxygen plasma-treated silicon or glass substrates, and the substrates were allowed to dry under ambient conditions for 1 hour. After drying, the substrates were rinsed with water and $70 \%(\mathrm{v} / \mathrm{v})$ ethanol.

SDS-PAGE/Western blot: The purity of filtered curli nanofibers was assessed using SDSPAGE. Curli fibers scrapped off from filter membranes were disassembled by dissolving them in a 1:1 (v/v) HFIP/TFA mixture, and incubating with sonication until the solution turned clear. After evaporating the solvent, the samples were resuspended in DI water and loading buffer. Western blotting was used to confirm the presence of HisTags on curli fibers after filtration. Samples were run on a NuPAGE Novex 4-12\% Bis-Tris gel and transferred on an iBlot PVDF membrane (Invitrogen). After blocking with $5 \%$ milk in TBST, the membrane was treated with a monoclonal mouse anti-His antibody HRP conjugate (ThermoFischer). Chemiluminescence was detected using a FluorChem ${ }^{\mathrm{TM}}$ M system (Protein Simple). 
Electron microscopy: Scanning electron microscopy (SEM) samples were prepared by washing the filter membranes with deposited curli nanofibers with $0.1 \mathrm{M}$ sodium cacodylate buffer, and fixing with $2 \%(\mathrm{~m} / \mathrm{v})$ glutaraldehyde and $2 \%(\mathrm{~m} / \mathrm{v})$ paraformaldehyde for 2 hours at room temperature. The membranes were then washed in water, and the solvent was gradually exchanged to ethanol with an increasing ethanol 15 minuteincubation step gradient $(25 \%, 50 \%$, $75 \%$ and $100 \%(\mathrm{v} / \mathrm{v})$ ethanol). The membranes were dried in a critical point dryer and sputtered until they were coated in a $5 \mathrm{~nm}$ layer of Pt/Pd. Imaging was performed using a Zeiss Ultra 55 Field Emission SEM.

Confocal microscopy: Curli nanofibers deposited on membranes were stained with thioflavin $T$ (ThT) (20 mM solution in water) for $1 \mathrm{~h}$, and DNA with SYTO 59 Red Fluorescent Nucleic Acid Stain ( $5 \mu \mathrm{M}$ solution in water) for $30 \mathrm{~min}$. A Leica SP5 X MP Inverted Confocal Microscope was used for imaging.

Fourier-Transformed Infrared Spectroscopy (FTIR): FTIR was used to evaluate the secondary structure of $\mathrm{Csg}$ A in lyophilized curli fibers obtained via filtration and in free-standing curli films. The measurements were performed with a Brucker Vertex 70 Spectrometer. Attenuated total reflection (ATR) spectra in the $1550-1750 \mathrm{~cm}^{-1}$ range were obtained with a 1 $\mathrm{cm}^{-1}$ resolution. Curve fitting was performed using the OPUS software.

Mass spectrometry: Bands were cut from Coomassie blue-stained SDS-PAGE gels and digested with trypsin. Microcapillary electrospray LC/MS/MS analysis was performed at the Taplin Mass Spectroscopy Facility using an Orbitrap mass spectrometer (Thermo Scientific).

\section{RESULTS AND DISCUSSION.}


In order to prevent chromosomally expressed curli proteins from confounding our results, we made use of an E. coli strain from which all of the curli genes ( $\operatorname{csg} B A C, \operatorname{csg} D E F G)$ had been deleted, PQN4. PQN4 was derived from a strain that does not produce any other extracellular polymers (e.g. flagella, pili, cellulose).$^{15}$ This strain was complemented by a plasmid encoding a subset of the genes necessary for curli production and secretion $(\operatorname{csg} A C E F G)$, without the presence of $\operatorname{cgs} D$ and $\operatorname{csg} B . \operatorname{csg} D$ was not included because its primary role is regulating the expression of the curli genes through transcriptional repression, and was therefore unnecessary for our purposes. The gene product for $\operatorname{csg} B$ is a membrane bound protein $(\mathrm{CsgB})$ whose main roles are to initiate amyloid formation at the cell surface and anchor curli fibers to the cell. Therefore, $\operatorname{csg} B$ was not included in the plasmid so that secreted and assembled CsgA would remain untethered to the bacterial surface, thereby simplifying purification. As shown in Figure 1, CsgA proteins overexpressed in the absence of CsgB spontaneously assemble into large aggregates in solution, while they remain more dispersed and anchored to the bacteria in the presence of $\mathrm{CsgB}$. Although $\mathrm{CsgB}$ is known to act as a nucleator for the polymerization of CsgA in vivo, ${ }^{33}$ it has also been demonstrated that CsgA can self-polymerize in vitro in the absence of $\mathrm{CsgB}^{34}$ The absence of $\mathrm{CsgB}$ also facilitates lateral aggregation of fibers during the selfassembly process, which would otherwise be prevented if fibers were only nucleated at distinct, spatially separated sites on the cell surface, i.e. in the presence of $\mathrm{CsgB}$. We decided to take advantage of this phenomenon to design our novel curli purification process based on filtration. The curli fiber aggregates, which were tens to hundred of microns in size, allowed for their sizedependent separation from bacteria and other small molecules or debris. 
A.

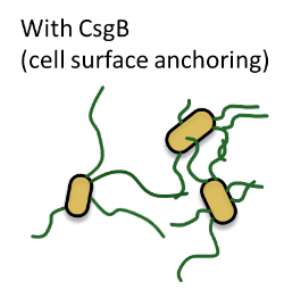

Without CsgB (no cell surface anchoring)

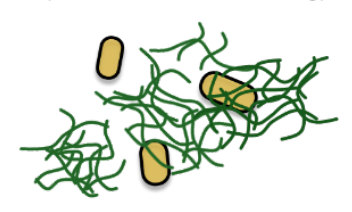

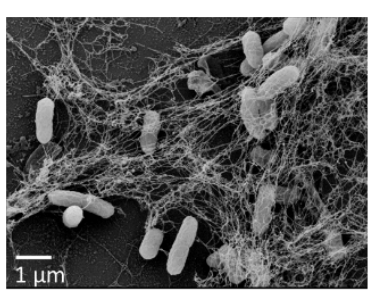

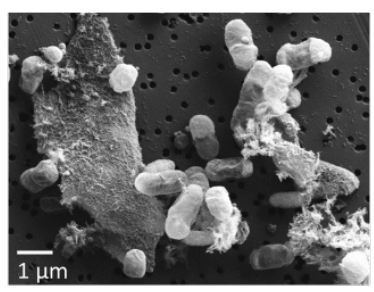

B.

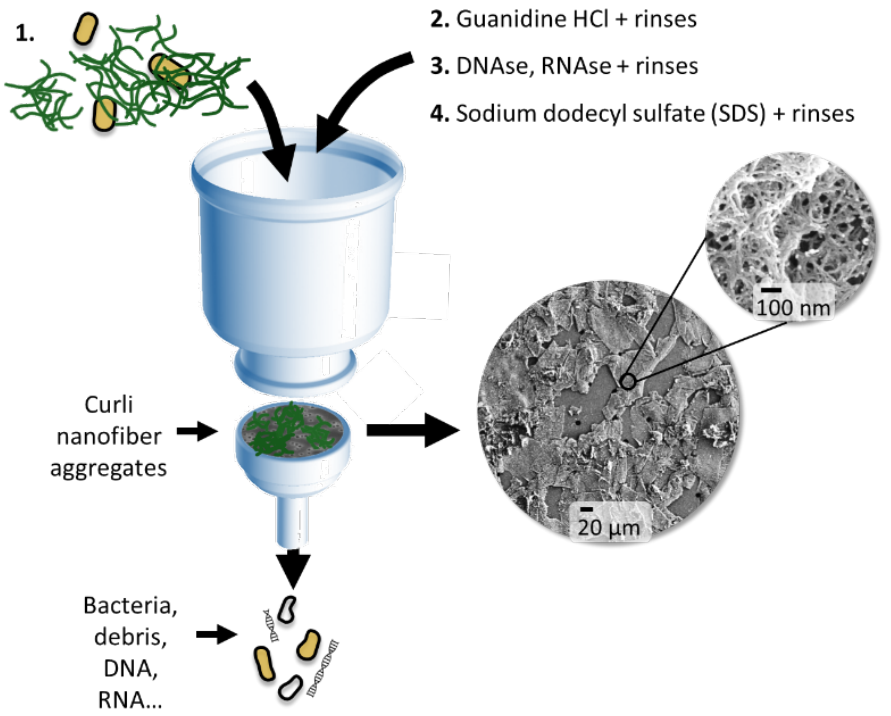

Figure 1. Size-dependent separation of curli nanofibers is possible due to the formation of large curli aggregates in the absence of the curli nucleator protein CsgB. A. Morphological differences between filtered bacterial cultures producing CsgA expressed along with (top) or without (bottom) CsgB, visualized with SEM prior to any purification. B. Schematic of the filtration purification process to trap large curli nanofiber aggregates on filter membranes and remove any impurities or cell debris via filtration. SEM images of large purified curli aggregates on a $10 \mu \mathrm{m}$ polycarbonate membrane are shown.

After overnight induction of bacterial culture to produce extracellular CsgA, $\mathrm{GdmCl}$ was added to the culture to a final concentration of $0.8 \mathrm{M}, 45$ to 120 minute prior to filtration. The mixture was then filtered through a polycarbonate membrane with $10 \mu \mathrm{m}$ pores using vacuum filtration, as illustrated in Scheme 1. We found that this first incubation with $\mathrm{GdmCl}$, allowed for significantly larger culture volumes to be filtered without clogging the membranes. Since GdmCl is a strong chaotropic agent that can disrupt the integrity of the bacterial cell membranes, we speculate that this was due to partial cell lysis. ${ }^{35}$ Next, three sequential incubations, each followed by water rinses, were performed directly on the filter membranes: 1) $8 \mathrm{M} \mathrm{GdmCl}$ was used to lyse any remaining bacterial cells and remove non-specifically bound proteins from the curli fibers, 2) a solution of a nuclease with DNAse and RNase activity was used to digest nucleic acids that tend to bind to curli fibers, ${ }^{36}$ and 3) incubation with SDS allowed for 
delamination of curli aggregates from the membrane and easy collection of the purified fibers with a spatula.

We used an amyloid-specific dye, Congo Red, in order to visually monitor the retention of assembled curli fibers during the filtration process. After the initial filtration of the bacterial culture, followed by water rinses to remove any non-specifically adsorbed dye, a bright red color was observed for membranes containing wild-type curli nanofibers (Figure 2A). Although the color was mostly retained during the sequential purification steps, we did observe a slight decrease in dye intensity, which could be attributed to a loss or partial degradation of curli fibers material, or to the removal of other aggregated proteins or cellular debris that binds Congo Red non-specifically. A negative control with bacteria expressing only maltose binding protein did not show any Congo Red retention, as expected.

A.

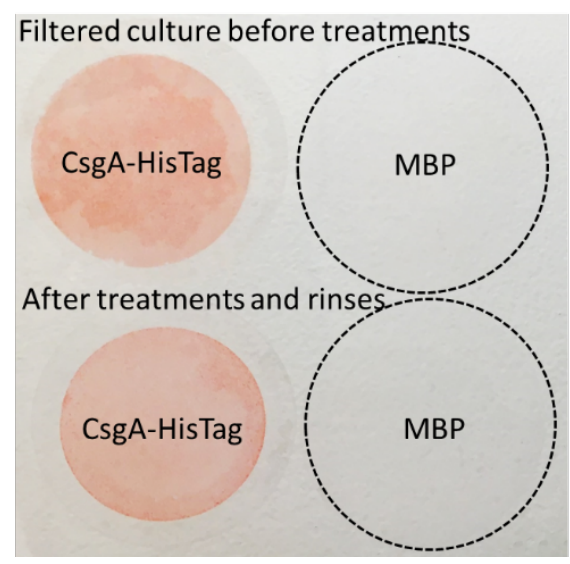

B.

Collection of purified fibers

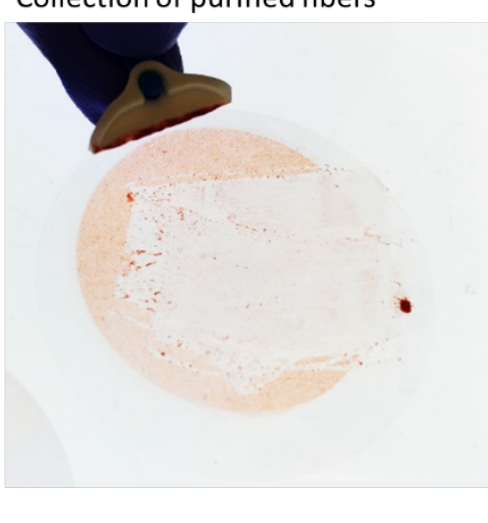

Figure 2. Congo red staining shows the presence of curli fibers on filter membranes. A. Congo red staining of membranes after filtration of the bacterial cultures, without additional treatment (top) and with full treatment with $\mathrm{GdmCl}$, nuclease and SDS (bottom) for bacteria producing wild-type curli fibers (CsgA) or MBP as a negative control. B. After the SDS treatment, filtered curli fibers can easily be scraped off of the filters to obtain semi-pure protein.

Some applications for amyloid-based materials, such as water decontamination, ${ }^{8}$ could directly make use of curli nanofiber films deposited on filter membranes. However, other 
applications would benefit from the removal of nanofibers aggregates from the membranes, or even solubilization and disassembly of the fibers into monomeric CsgA subunits. After the full purification sequence, curli nanofibers can be simply scrapped off of the membrane surface using a spatula (Figure 2B). In addition, they can be solubilized, disassembled using a 1:1 HFIP/TFA mixture and reassembled in water or buffer. After trying several solvents to facilitate removal of the curli fibers from the membrane, SDS incubation was selected as the last step in the purification process because it allows for the delamination of the curli nanofiber films from the membranes. If the SDS incubation step is replaced with an incubation with bases or acids, the yield of nanofibers decreases significantly after scraping (Figure 3A). Surprisingly, formic acid did not facilitate the removal of the fibers from the membranes, even though it is known to disassemble curli fibers. ${ }^{21}$

To investigate the impact of the $\mathrm{GdmCl}$ incubation step on purity, curli nanofibers were collected from filter membranes after being treated with varying concentrations of $\mathrm{GdmCl}$ from 0 to $8 \mathrm{M}$. The number and concentration of impurities decreases as the concentration of $\mathrm{GdmCl}$ increased (Figure 3B), although some impurities persisted even at the highest $\mathrm{GdmCl}$ concentrations. The main impurity observed by Coomassie staining of the gels, at $\sim 14 \mathrm{kDa}$ was identified by mass spectrometry as partially degraded CsgA (see Table S2), and other impurities correspond to a mixture of other E. coli proteins. Densitometry analysis of the Coomassie gel bands from the purification using $8 \mathrm{M} \mathrm{GdmCl}$ indicates that $\mathrm{CsgA}$ proteins make up $\sim 80 \%$ of the total protein content of the material, with $15 \%$ of this in the degraded form. If the domains that are fused to CsgA are prone to denaturation, lower concentrations of $\mathrm{GdmCl}$ could be used, at the expense of protein purity. Relatively pure $\mathrm{CsgA}$ can be obtained at $\mathrm{GdmCl}$ concentrations as low as 2 or $4 \mathrm{M}$, which could allow for the purification of a wide variety of genetically 
engineered CsgA fusions. While concentrations of $\mathrm{GdmCl}$ in the range of 4 to $8 \mathrm{M}$ are normally known to cause partial or full protein denaturation, ${ }^{37} 38$ curli nanofibers are highly resistant to denaturing agents and solvents ${ }^{22} 16$ and likely create a protective mesh that could prevent or reduce denaturation of protein domains fused to $\mathrm{Csg} A$, as observed previously for bio-active molecules like enzymes immobilized onto protein scaffolds. ${ }^{39} 17$ If desired, further purification of the curli fibers can be achieved via dialysis, to remove any small residual proteins or peptides (Figure 3C). The presence of CsgA in the final purified product was confirmed using Western blot (Figure 3D).

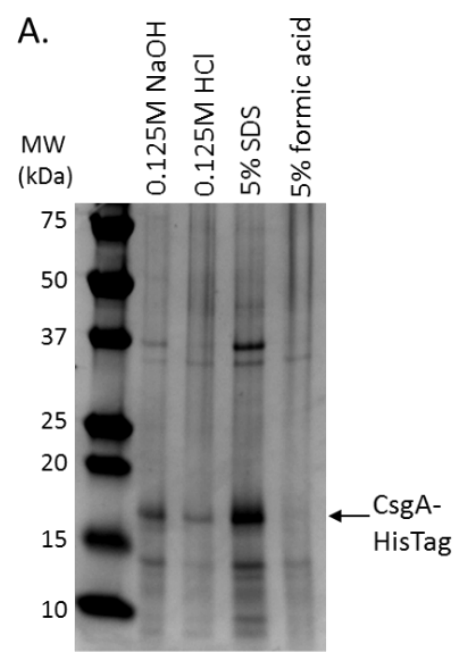

B.

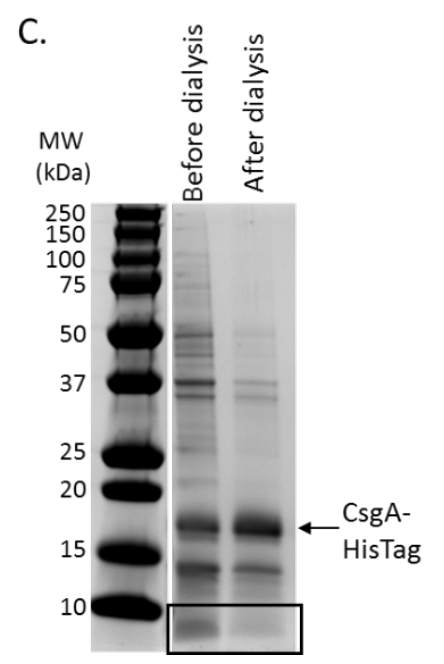

D.

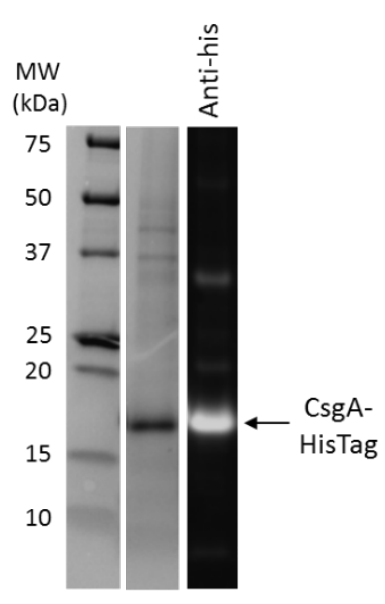


Figure 3. SDS-PAGE analysis of CsgA purity after treatment of filtered bacterial cultures with $\mathrm{GdmCl}$ and subsequent washing steps, followed by removal from membranes. A. Several final washing steps $\mathrm{NaOH}, \mathrm{HCl}$, SDS, formic acid) were tested, resulting varying CsgA purities. The $\mathrm{GdmCl}$ wash was fixed at $8 \mathrm{M}$. SDS was selected for further experiments because it best facilitated removal of fibers from the membrane. B. Curli nanofiber purity as a function of $\mathrm{GdmCl}$ concentration used for the on-filter incubation step. C. Further purification of isolated curli fibers by dialysis in a 6-8 kDa MWCO membrane led to removal of some low MW impurities (black box). CsgA-HisTag appears at its expected molecular weight of $16.7 \mathrm{kDa}$. D. Curli nanofibers, composed of CsgA-HisTag and purified by filtration, visualized with SDS-PAGE/Coomassie (left) and detected by Western blot (right) using an anti-His antibody.

Scanning electron microscopy (SEM) was used to visualize the curli thin films directly on filter membranes after fixation with glutaraldehyde and paraformaldehyde to preserve their native morphology (Figure 4). Without any $\mathrm{GdmCl}$ treatment, several bacteria were present on the surface of the curli aggregates. The vast majority of bacteria were removed by treating the cultures with $0.8 \mathrm{M} \mathrm{GdmCl}$ prior to filtration, but a few intact cell bodies could still be identified. The most effective step for removing bacterial cells was the $8 \mathrm{M} \mathrm{GdmCl} \mathrm{rinse} \mathrm{after} \mathrm{filtration} \mathrm{of}$ the culture (Figure 3B). After both the $\mathrm{GdmCl}$ pre-treatment, and the $8 \mathrm{M} \mathrm{GdmCl}$ wash step, several empty spaces in the film appeared, which were presumably occupied by bacteria previously. In all cases, nanofibrous features remained visible after $\mathrm{GdmCl}$ incubations (Figure 4, insets), but the fibers tended to aggregate more with exposure to $\mathrm{GdmCl}$. 
A.

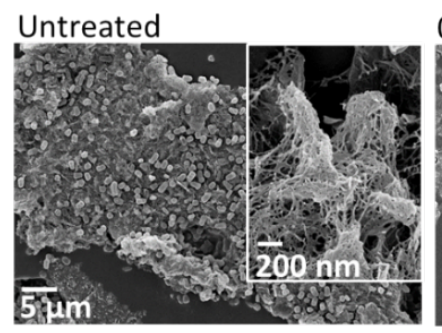

$0.8 \mathrm{M} \mathrm{GdmCl}$ pre-filtration
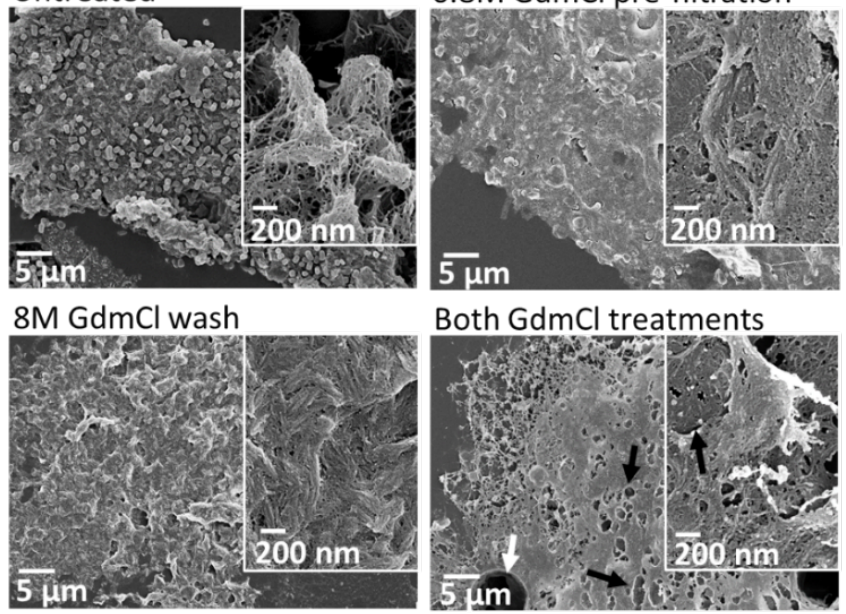

Both $\mathrm{GdmCl}$ treatments

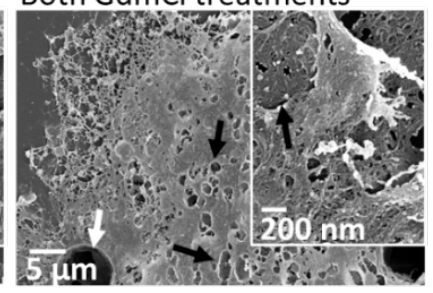

B.

\section{Curli nanofibers on filter}
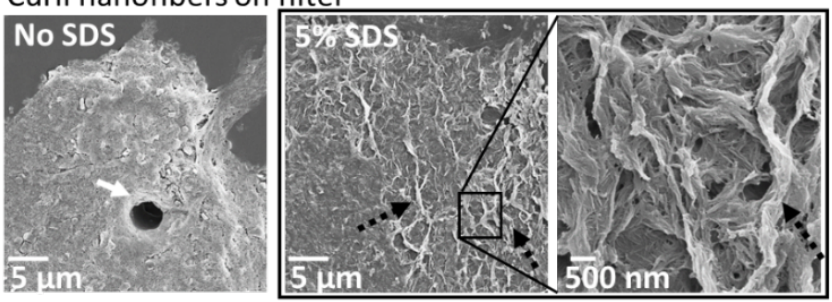

Filter after scraping
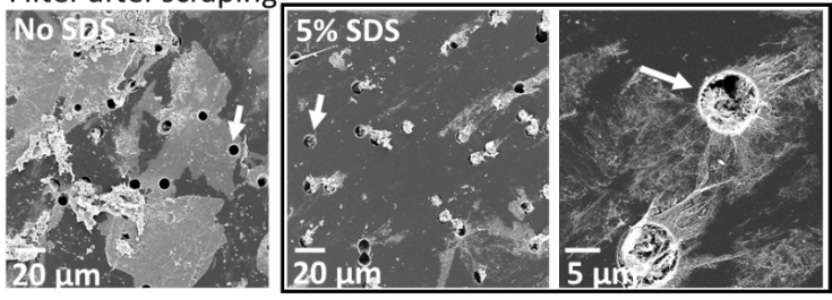

Figure 4. $\mathrm{GdmCl}$ and SDS treatments allow for purification and delamination of the curli nanofiber films. Scanning electron microscopy images after various treatments. A. $\mathrm{GdmCl}$ effectively removes cells from the curli fibers, leaving behind voids where cells used to be (black arrows). The aggregation state of the fibers also appears to be affected. $10 \mu \mathrm{m}$ pores of the membrane are also indicated (white arrows). B. SDS treatment after cell removal causes the film to wrinkle (black dashed arrows) and makes removal straightforward from the membrane surface.

We also obtained SEM images of filtered films before and after SDS treatment in an effort to understand why SDS made removal so much easier compared to other treatments (Figure 3B). SDS treatment caused the film to wrinkle and partially delaminate from the surface of the membrane. We then attempted to scrape off curli nanofibers from filters treated or not with SDS. Without SDS incubation, it was impossible to remove the nanofibers from the surface 
and the aggregates remained intact, deposited on the membrane. In contrast, very little material remained on the surface of filters treated with SDS after scraping. Interestingly, surface wrinkling is also used in microbiological studies as a phenotypic marker for the presence of curli fibers in biofilms. ${ }^{40} 41$

The purification of curli nanofibers from DNA was further confirmed by confocal microscopy using SYTO 59 Red Fluorescent Nucleic Acid Stain to stain DNA and RNA. After treatment with nuclease, a significant decrease in the amount of nucleic acids was observed within the curli fiber mesh, and only a background fluorescence signal remained. The presence of the curli fibers on the membrane through the process was also confirmed by Thioflavin $\mathrm{T}$ (ThT) staining (Figure S1).

After determining that this filtration scheme was suitable for the purification of tag-less, wild-type CsgA, we sought to do a similar demonstration for amyloid fibers displaying fused functional domains, thereby providing an entry point to genetically engineered protein-based materials. We have found the SpyTag-SpyCatcher conjugation scheme ${ }^{31}$ to be highly effective for immobilization of proteins to assembled curli fibers, but we wanted to confirm that the 13 amino acid SpyTag domain was active after exposure to the rigorous filtration protocol. Therefore, we treated filtered curli fibers displaying SpyTag to the washing protocol, then exposed them to a Venus-SpyCatcher fusion protein. As expected, filters coated with SpyTag fibers retained the Venus-SpyCatcher protein after washing, while those coated with CsgA-His fibers did not, suggesting that the fluorescent protein capture was specific to the SpyTagSpyCatcher interaction. Filters treated with MBP-expressing bacteria and bare filters also did not trap the Venus-SpyCatcher chimera (Figure 5A). In addition to short peptide tags, we also sought to demonstrate the utility of our approach to display longer functional protein sequences. 
Accordingly, we constructed genes encoding for CsgA-SpyCatcher fusions. There is some precedent for the fusion of large protein domains to CsgA without disrupting the secretion and assembly mechanism, however, secretion efficiency is difficult to predict a priori based on size and sequence alone. We found that CsgA-SpyCatcher fusions could be expressed and purified as assembled curli fibers using the filtration protocol. (Figure S2). Remarkably, the SpyCatcher domain also remained active and could covalently capture fluorescent Venus-SpyTag proteins (Figure 5B), even after exposure to the highly denaturing filtration protocol. These results suggest that engineered amyloid fibers that vary widely in sequence length could be purified via filtration and remain functional.

A.

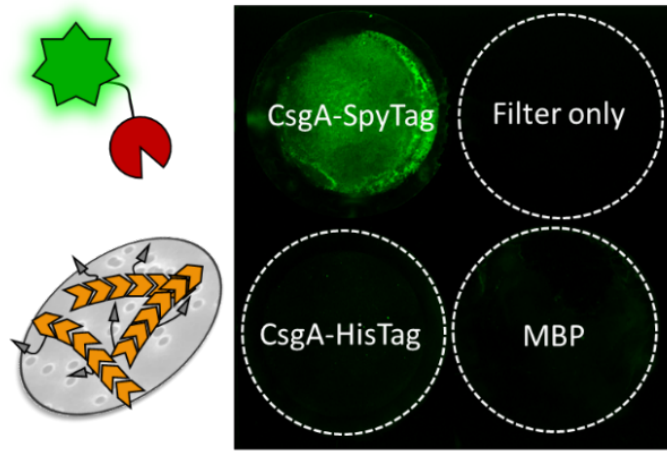

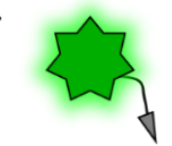
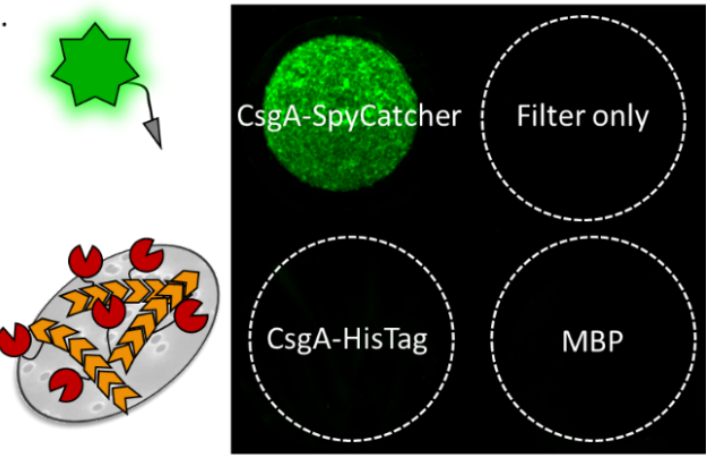

\section{$\sum \operatorname{CsgA} \quad \Delta \operatorname{SpyTag}$}

SpyCatcher

Flexible linker

Venus

Figure 5. Engineered curli nanofibers displaying small tags or larger protein domains remain active after purification. A. Filter membranes coated with SpyTag-displaying curli fibers can bind Venus-SpyCatcher after purification. B. Filter membranes coated with SpyCatcher-displaying curli fibers can bind Venus-SpyTag after purification. Cells expressing CsgA-HisTag, and MBP were subjected to the same filtration protocol and exposed to the corresponding Venus fusion proteins. Bare polycarbonate filters incubated with Venus fusion proteins also showed no fluorescent signal.

In order to estimate the yield of pure curli nanofibers using our protocol, we weighed the purified fibers obtained from $40 \mathrm{~mL}$ of induced bacterial culture filtered through a single $47 \mathrm{~mm}$ diameter membrane after scraping them off with a spatula (Figure S3). A representative experiment yielded 30 to $45 \mathrm{mg}$ of CsgA-HisTag per liter of culture. The limiting factor in the 
scalability of this protocol was clogging of the filter lyophilized purified fibers. By increasing the surface area of the filter membrane, scaling up the filtration purification process becomes trivial and can allow for large-scale production of genetically engineered curli nanofibers with a desired function. As a demonstration and first step towards scaling up our process, we used a $142 \mathrm{~mm}$ diameter filter membrane to filter up to 10 times more culture volume (Figure S3). Filtering large volumes of culture can allow for easily producing 100s of milligrams of purified curli materials, which translates to simple, low-cost and rapid scalability. In comparison with affinity purification methods for curli fibers, ${ }^{22}$ the mass of amyloid materials obtained by filtration is one to two orders of magnitude greater. Coupled with further optimization of the biosynthetic machinery, and the use of batch fermentation, this technique could yield significantly more quantities of pure protein.

In addition to isolating recombinant protein fibers, the filtration protocol also proved to be useful as a fabrication method for producing self-standing, macroscopic, amyloid-based films (Figure 6). While the production of functional amyloid-based materials via vacuum filtration was previously accomplished by forming composites of amyloids with nanomaterials on filter membranes, ${ }^{26} 27282930$ our method adds another layer of functionality that can be achieved through genetic engineering. In order to produce genetically engineered materials with our system, filter membrane-supported curli fibers were crosslinked using a mixture of glutaraldehyde and paraformaldehyde. Then, the underlying polycarbonate membrane was dissolved in a dichloromethane bath (Figure S4). In less than a minute, the polycarbonate membrane fully dissolved, and the curli film began to float in solution. A Teflon membrane was used to collect the free-standing curli film, and, after drying, the curli film could easily be peeled off the Teflon support. The resulting thin film was transparent and flexible (Figure 6B, C). 
Remarkably, even after crosslinking and immersion in organic solvent, the activity of the displayed SpyTag fusion was maintained (Figure 6D).
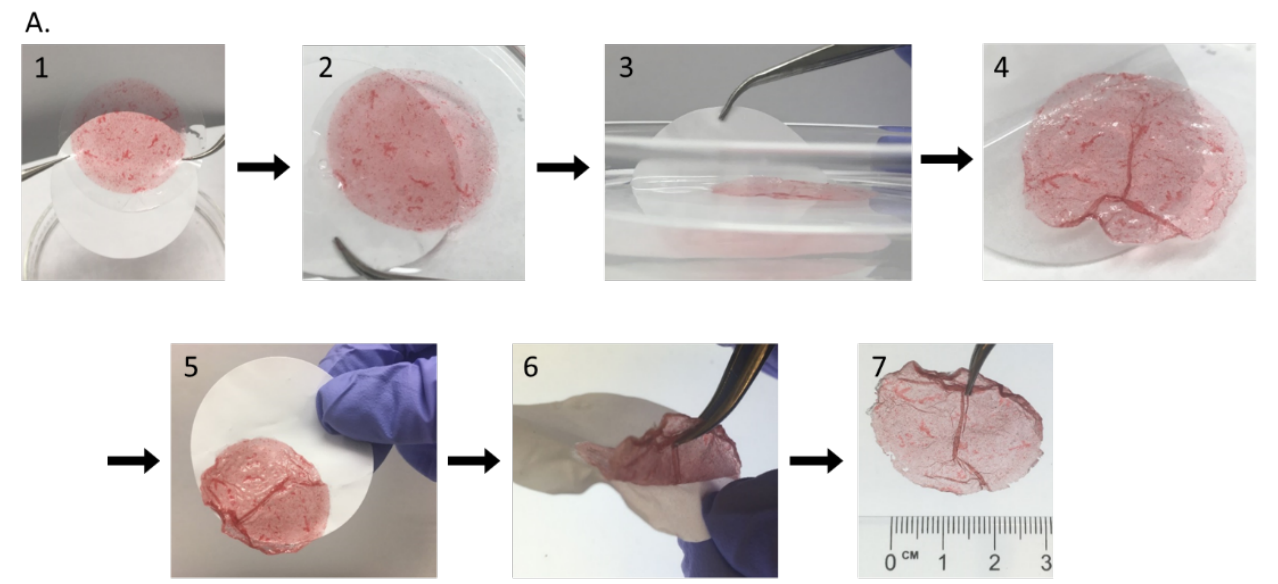

B.

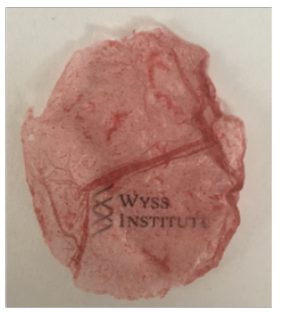

E.

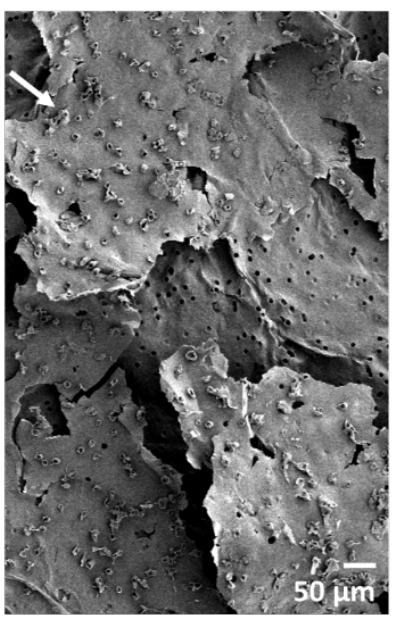

C.

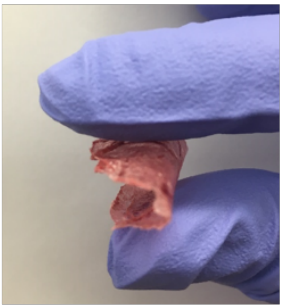

D.
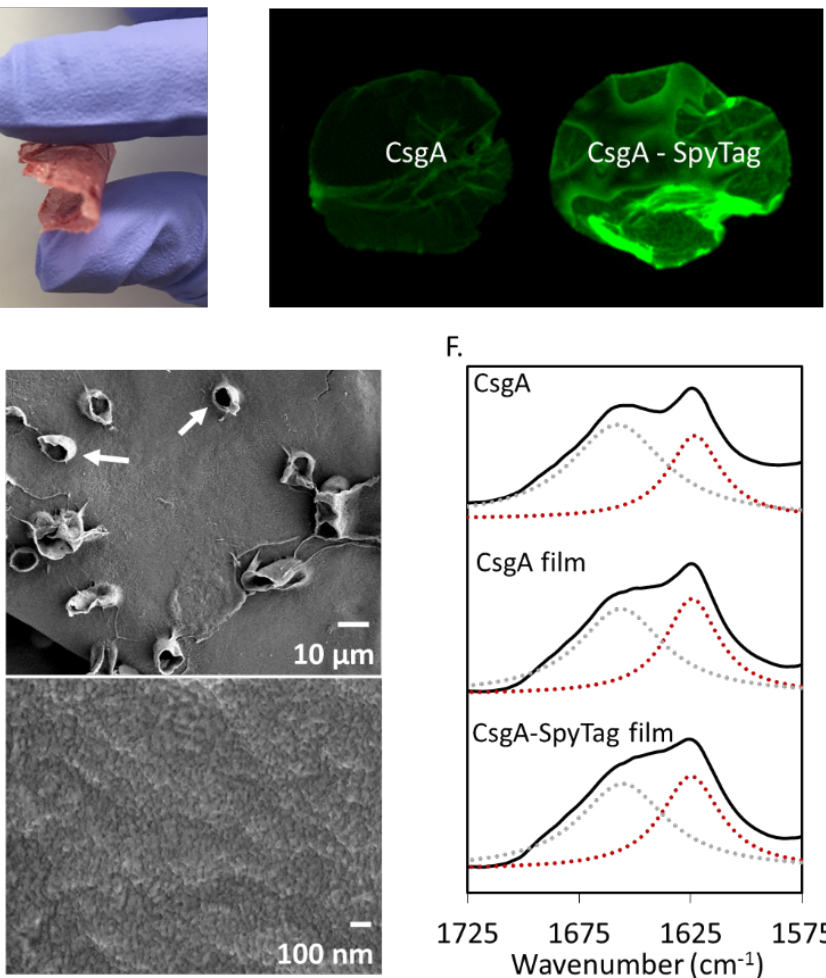

F.

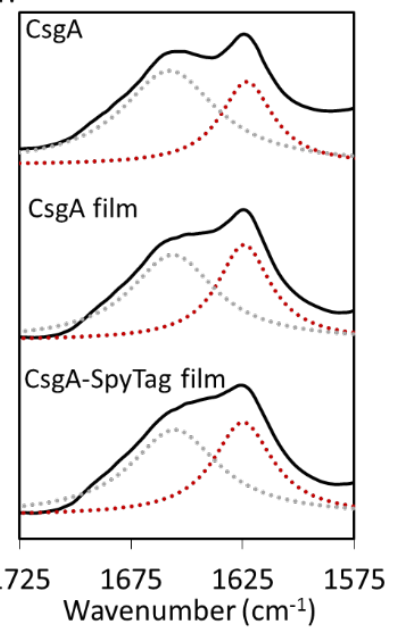

Figure 6. Free-standing curli fiber thin films fabricated via filtration. A. The fabrication protocol begins with purified, crosslinked fibers on a polycarbonate filter membrane. A Teflon membrane is placed on top of the filtered curli fibers (1), and the sandwiched fibers are immersed in DCM (2). Within seconds, the polycarbonate membrane is dissolved and the curli film begins to float (3). The Teflon membrane is used to collect the floating film (4). The curli film is dried on the Teflon membrane (5), after which it can be peeled off (6) to yield an intact macroscopic 
thin film composed of assembled curli fibers. The thin films are transparent (B), flexible (C), and display functional peptide tags that remain active throughout the process (D). D. Free-standing films, composed of either CsgA-His or CsgA-SpyTag were exposed to Venus-SpyCatcher, washed, and visualized with fluorescence. E. SEM of dried, freestanding curli thin films. The pore pattern of the filter membrane is reproduced in the film, with extrusions (white arrows) where portions of the film were sucked through the filter pore. F. FTIR analyses of the amide I of crosslinked films compared to that of scraped CsgA powder (black traces). Spectra were reconstituted by a linear sum of two amide-related peaks: indicative of the presence of $\beta$-sheets (red traces) and disordered or aggregated structures (grey traces).

The morphology and secondary protein structure of the films were investigated. SEM was used to visualize free-standing films that had been air-dried on the bench top and revealed the presence of large overlapping sheets of material, with dimensions corresponding approximately to the size of the curli aggregates previously observed on polycarbonate membranes after filtration (Figure 6E). Other images show that pores from the polycarbonate were imprinted in the curli structure, and that portions of the curli fiber mats that were pulled into the pores during filtration formed extruded cylindrical structures. At higher magnifications, a tightly interconnected nanoporous structure can be observed, likely corresponding to curli fibers that collapsed onto each other due to drying. FTIR further confirmed that the freestanding films were composed of material with secondary structure similar to lyophilized curli fibers scraped from filter membranes. Figure $6 \mathrm{~F}$ shows the amide I peak spectra for lyophilized purified curli, and for free-standing films of CsgA and CsgA-SpyTag. In all cases the curves can be fitted with two Lorentz peaks corresponding to $\beta$-sheet folded proteins (around $1624 \mathrm{~cm}^{-1}$ ) and to disordered or aggregated structures (around $1650 \mathrm{~cm}^{-1}$ ). ${ }^{42}{ }^{43}$ With preserved secondary structure and binding activity, the free-standing curli fiber films could be used to bind a variety of functional proteins, enzymes or molecules using the SpyTag-SpyCatcher system for a wide range of applications.

Finally, we showed that the fibers collected via filtration can be dissolved in an HFIP/TFA mixture with sonication until the solution turns clear, and that thin films of fibers can 
be directly obtained by dropcasting the fiber solution onto substrates (Figure 7A). The thin films can be stained with Congo Red, showing the presence of assembled amyloid fibers. The thin films can also be delaminated from the substrates after rinsing with surfactants like SDS, and then re-used to form other films or materials. This cycle can be repeated due to the properties of curli fibers to diassemble on-demand in certain solvents, and to reassemble when these solvents evaporate. Upon drying, we confirmed the reassembly of the fibers by SEM (Figure 7B), which shows a highly porous and fibrous film cross-section. Such recycling of curli nanofibers could greatly reduce the production cost of curli-based materials that require different material shapes or coating geometries.

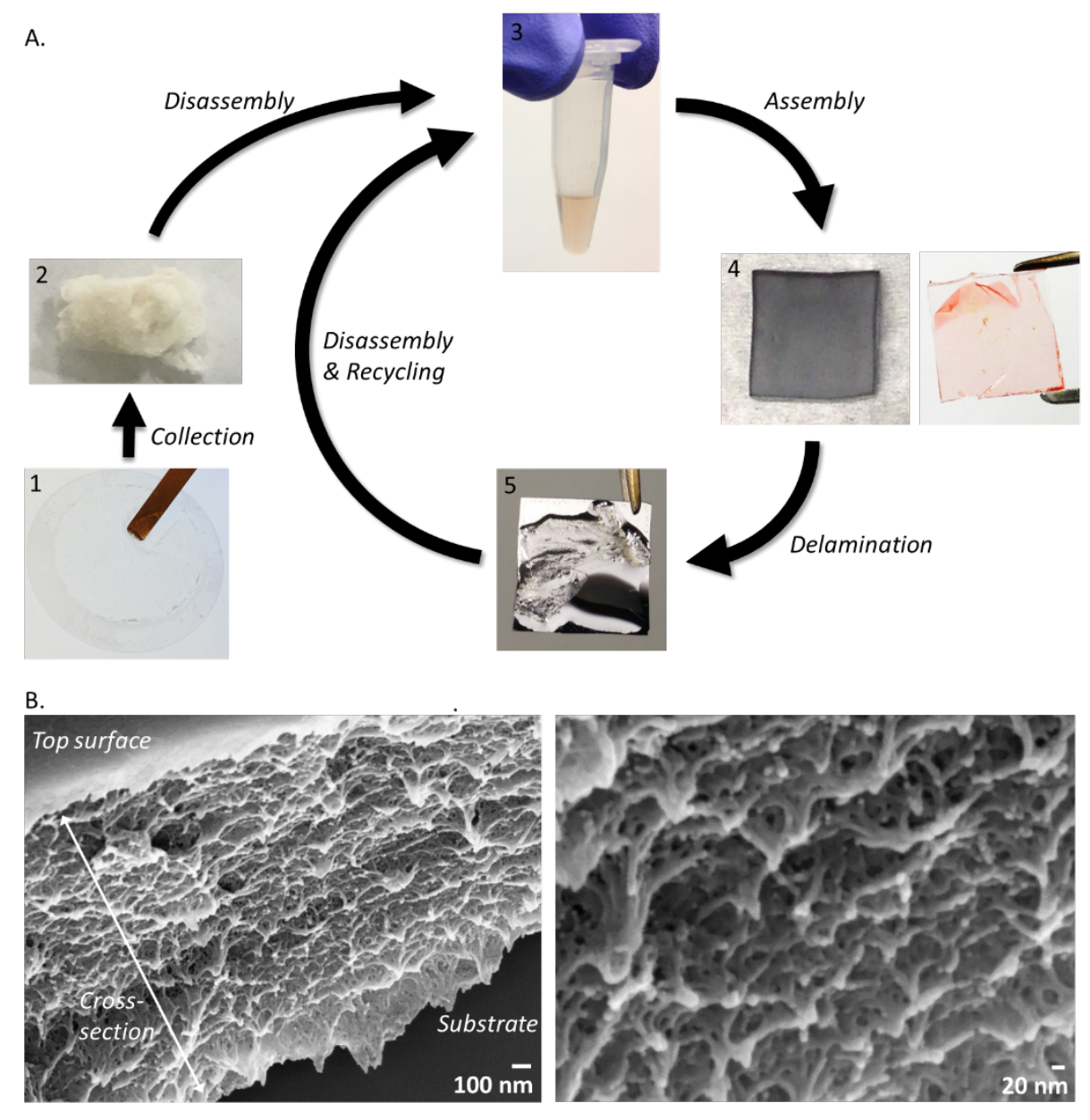


Figure 7. Formation and recycling of thin films fabricated from filtered curli fibers. A. Curli fibers scraped from filter membranes (1) and dried (2) are disassembled using HFIP/TFA, producing a clear solution (3). The solution can be dropcast onto various substrates (4) including silicon (left) and glass (right, after Congo Red staining) to form thin films. After rinsing with SDS, the curli fiber film delaminates from the substrate to form a flexible free-standing film (5), which can then be recycled by disassembling the fibers again with HFIP/TFA to fabricate a new curli-based material. Substrates shown have $1 \mathrm{~cm}$ by $1 \mathrm{~cm}$ dimensions. B. Thin curli films formed by dropcasting a $15 \mathrm{mg} / \mathrm{mL}$ solution of curli in HFIP and TFA are fibrous and porous, as visualized by SEM. The cross-section of the film is shown (left) and a higher magnification image (right) allows for fiber visualization.

\section{CONCLUSIONS.}

We have demonstrated a streamlined and scalable purification procedure for recombinantly produced amyloid proteins. The procedure, which relies on filtration followed by washes in chaotropic solutions, is particularly suited to the purification of the fibrous curli proteins of the $E$. coli biofilm matrix, due to their propensity to self-assemble into extracellular micro-scale aggregates in the absence of $\mathrm{CsgB}$, and their remarkable resistance to denaturing agents. However, other fibrous proteins that exhibit similar properties may be compatible with this general scheme, depending on their resistance to denaturants in the assembled state. Overall, the method we demonstrate enables the isolation of pure, assembled curli fibers, and can easily be scaled to isolate 100 s of mg quantities of protein with easily accessible equipment (Figure 8 ). Additionally, the filtered fibers with highly customizable pendant sequences can be used directly on the filter membrane for affinity purification/removal applications. 


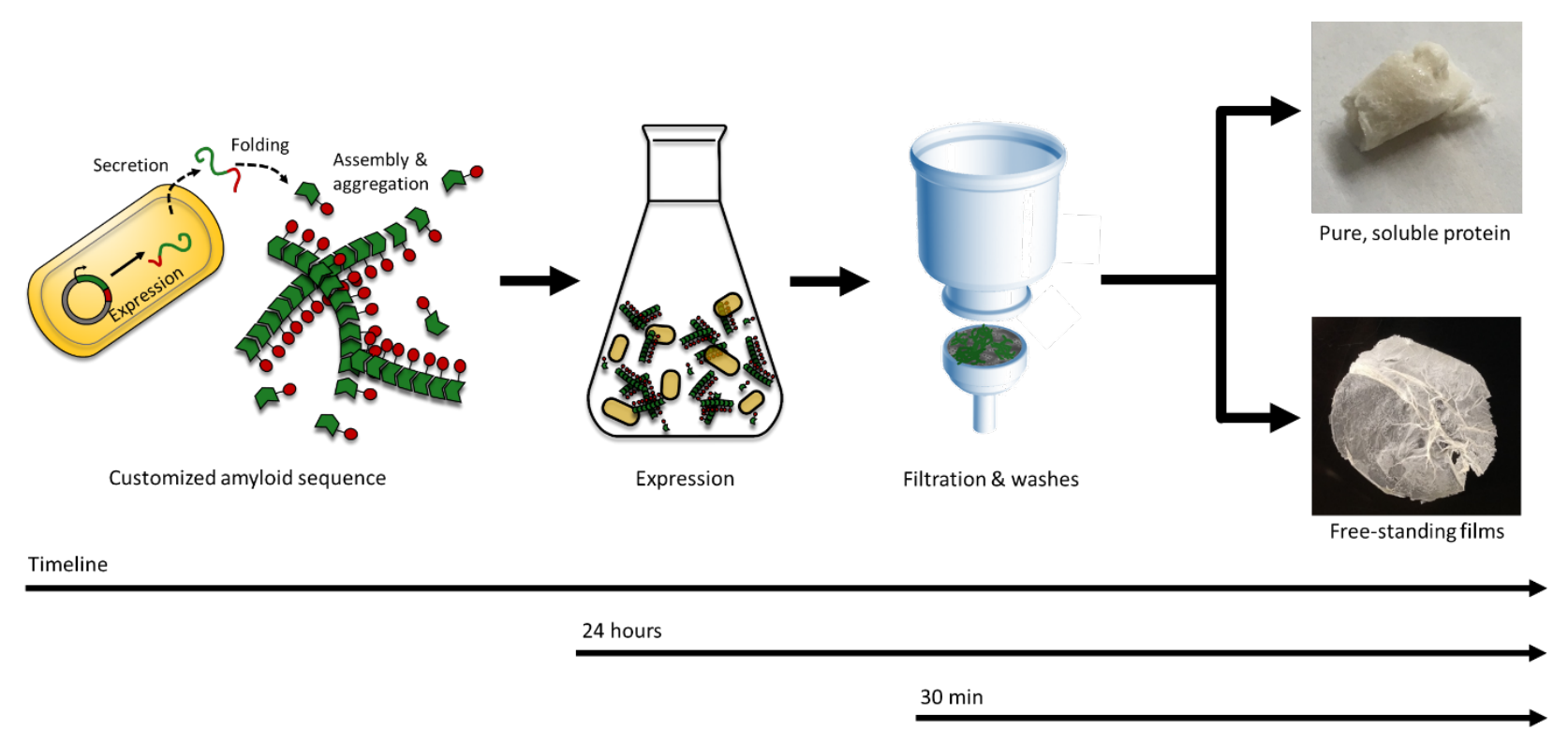

Figure 8. Workflow for fabricating genetically engineered curli-based macroscopic materials based on fast and simple processing steps. Cloning steps and transformation of engineered bacteria requires with a customized CsgA variant requires two to three days. Growth and expression of assembled curli fibers can be accomplished in 24 hours, after which filtration and washes are carried out in 30 minutes, producing enough pure protein to assemble various materials like free-standing thin films.

In comparison to other purification techniques, which usually require several time consuming and low-yielding steps, this method is likely to be an easier and cheaper way to obtain sufficient quantities of recombinant protein to fabricate macroscopic materials, without sacrificing molecular-level control over structure. The method does not require any purification tag, and is compatible with curli variants with widely varying structures. This purification protocol can be coupled with several established techniques to create integrated materials fabrication protocols with these easily engineered proteins. We anticipate that this simple workflow can be used to create materials for a wide range of applications for which sequence customizability is critical.

\section{ASSOCIATED CONTENT}

Supporting Information. C-terminal sequence modifications of CsgA, Mass spectrometry analysis, Confocal Microscopy imaging, Congo Red dye binding on cell pellets, Scale-up of 
filtration process, Dissolution of polycarbonate membranes. The following files are available free of charge.

\section{AUTHOR INFORMATION}

\section{Corresponding Author}

*Email : neel.joshi@wyss.harvard.edu, Phone : 617-432-7730

\section{Author Contributions}

N.-M.D.C., A.D.-T., P.K.R.T. and N.S.J. conceived the idea and designed the experiments. N.M.D.C., A.D.-T. and P.K.R.T. performed the filtration experiments, characterization, and data analysis. P.Q.N. performed the curli operon knockout in the PQN4 strain. The manuscript was written through contributions of all authors. All authors have given approval to the final version of the manuscript. ${ }^{\S}$ These authors contributed equally.

\section{ACKNOWLEDGMENT}

This work made use of shared facilities at the Harvard Center for Nanoscale Systems and at the Wyss Institute for Biologically Inspired Engineering at Harvard University. N.-M.D.C. gratefully acknowledges a Postdoctoral research fellowship from the Fonds de Recherche Nature et Technologies du Québec (FRQNT). This work was supported by NSF Grant 1410751 (DMR).

\section{REFERENCES}

1. Mankar, S.; Anoop, A.; Sen, S.; Maji, S. K., Nanomaterials: amyloids reflect their brighter side. Nano Reviews 2011, 2, 10.3402/nano.v2i0.6032. DOI: 10.3402/nano.v2i0.6032. 2. $\quad$ Rudra, J. S.; Mishra, S.; Chong, A. S.; Mitchell, R.; Nardin, E. H.; Nussenzweig, V.; Collier, J. H., Self-assembled peptide nanofibers raising durable antibody responses against a malaria epitope. Biomaterials 2012, 33 (27), 6476-6484. DOI:

10.1016/j.biomaterials.2012.05.041. 
3. Chen, A. Y.; Deng, Z.; Billings, A. N.; Seker, U. O. S.; Lu, Michelle Y.; Citorik, R. J.; Zakeri, B.; Lu, T. K., Synthesis and patterning of tunable multiscale materials with engineered cells. Nat Mater 2014, 13 (5), 515-523. DOI: 10.1038/nmat3912

4. Nguyen, P. Q.; Botyanszki, Z.; Tay, P. K. R.; Joshi, N. S., Programmable biofilm-based materials from engineered curli nanofibres. Nat Commun 2014, 5. DOI: 10.1038/ncomms5945.

5. Andersson, E. K.; Bengtsson, C.; Evans, M. L.; Chorell, E.; Sellstedt, M.; Lindgren, A. E. G.; Hufnagel, D. A.; Bhattacharya, M.; Tessier, P. M.; Wittung-Stafshede, P.; Almqvist, F.; Chapman, M. R., Modulation of Curli Assembly and Pellicle Biofilm Formation by Chemical and Protein Chaperones. Chemistry \& biology 2013, 20 (10), 1245-1254. DOI:

10.1016/j.chembiol.2013.07.017.

6. Schreck, J. S.; Yuan, J.-M., A Kinetic Study of Amyloid Formation: Fibril Growth and Length Distributions. The Journal of Physical Chemistry B 2013, 117 (21), 6574-6583. DOI: 10.1021/jp401586p.

7. Knowles, T. P. J.; Oppenheim, T. W.; Buell, A. K.; Chirgadze, D. Y.; Welland, M. E., Nanostructured films from hierarchical self-assembly of amyloidogenic proteins. Nat Nano 2010, 5 (3), 204-207. DOI:

8. Bolisetty, S.; Mezzenga, R., Amyloid-carbon hybrid membranes for universal water purification. Nat Nano 2016, 11 (4), 365-371. DOI: 10.1038/nnano.2015.310

9. Meier, C.; Lifincev, I.; Welland, M. E., Conducting Core-Shell Nanowires by Amyloid Nanofiber Templated Polymerization. Biomacromolecules 2015, 16 (2), 558-563. DOI:

10.1021/bm501618c.

10. Knowles, T. P. J.; Mezzenga, R., Amyloid Fibrils as Building Blocks for Natural and Artificial Functional Materials. Advanced Materials 2016, n/a-n/a. DOI:

10.1002/adma.201505961.

11. Meier, C.; Welland, M. E., Wet-Spinning of Amyloid Protein Nanofibers into Multifunctional High-Performance Biofibers. Biomacromolecules 2011, 12 (10), 3453-3459. DOI: $10.1021 / \mathrm{bm} 2005752$.

12. Jung, J.-M.; Savin, G.; Pouzot, M.; Schmitt, C.; Mezzenga, R., Structure of Heat-Induced $\beta$-Lactoglobulin Aggregates and their Complexes with Sodium-Dodecyl Sulfate.

Biomacromolecules 2008, 9 (9), 2477-2486. DOI: 10.1021/bm800502j.

13. Peralta, M. D. R.; Karsai, A.; Ngo, A.; Sierra, C.; Fong, K. T.; Hayre, N. R.; Mirzaee, N.; Ravikumar, K. M.; Kluber, A. J.; Chen, X.; Liu, G.-y.; Toney, M. D.; Singh, R. R.; Cox, D. L., Engineering Amyloid Fibrils from $\beta$-Solenoid Proteins for Biomaterials Applications. ACS Nano 2015, 9 (1), 449-463. DOI: 10.1021/nn5056089.

14. Woolfson, D. N.; Mahmoud, Z. N., More than just bare scaffolds: towards multicomponent and decorated fibrous biomaterials. Chemical Society Reviews 2010, 39 (9), 34643479. DOI: 10.1039/c0cs00032a.

15. Chapman, M. R.; Robinson, L. S.; Pinkner, J. S.; Roth, R.; Heuser, J.; Hammar, M.; Normark, S.; Hultgren, S. J., Role of Escherichia coli Curli Operons in Directing Amyloid Fiber Formation. Science 2002, 295 (5556), 851-855. 
16. Evans, M. L.; Chapman, M. R., Curli Biogenesis: Order out of Disorder. Biochimica et biophysica acta 2014, 1843 (8), 1551-1558. DOI: 10.1016/j.bbamcr.2013.09.010.

17. Botyanszki, Z.; Tay, P. K. R.; Nguyen, P. Q.; Nussbaumer, M. G.; Joshi, N. S., Engineered catalytic biofilms: Site-specific enzyme immobilization onto E. coli curli nanofibers. Biotechnology and Bioengineering 2015, 112 (10), 2016-2024. DOI: 10.1002/bit.25638.

18. Van Gerven, N.; Goyal, P.; Vandenbussche, G.; De Kerpel, M.; Jonckheere, W.; De Greve, H.; Remaut, H., Secretion and functional display of fusion proteins through the curli biogenesis pathway. Molecular Microbiology 2014, 91 (5), 1022-1035. DOI:

10.1111/mmi.12515.

19. Chen, A. Y.; Zhong, C.; Lu, T. K., Engineering Living Functional Materials. ACS Synthetic Biology 2015, 4 (1), 8-11. DOI: 10.1021/sb500113b.

20. Zhong, C.; Gurry, T.; Cheng, A. A.; Downey, J.; Deng, Z.; Stultz, C. M.; Lu, T. K., Strong underwater adhesives made by self-assembling multi-protein nanofibres. Nat Nano 2014, 9 (10), 858-866. DOI: 10.1038/nnano.2014.199

21. Zhou, Y.; Smith, D. R.; Hufnagel, D. A.; Chapman, M. R., Experimental Manipulation of the Microbial Functional Amyloid Called Curli. In Bacterial Cell Surfaces: Methods and Protocols, Delcour, H. A., Ed. Humana Press: Totowa, NJ, 2013; pp 53-75. DOI: 10.1007/978-162703-245-2_4.

22. Collinson, S. K.; Emödy, L.; Müller, K. H.; Trust, T. J.; Kay, W. W., Purification and characterization of thin, aggregative fimbriae from Salmonella enteritidis. Journal of Bacteriology 1991, 173 (15), 4773-4781.

23. Alberti, S.; Halfmann, R.; King, O.; Kapila, A.; Lindquist, S., A Systematic Survey Identifies Prions and Illuminates Sequence Features of Prionogenic Proteins. Cell 137 (1), 146158. DOI: 10.1016/j.cell.2009.02.044.

24. Sivanathan, V.; Hochschild, A., Generating extracellular amyloid aggregates using E. coli cells. Genes \& Development 2012, 26 (23), 2659-2667. DOI: 10.1101/gad.205310.112.

25. Sivanathan, V.; Hochschild, A., A bacterial export system for generating extracellular amyloid aggregates. Nat. Protocols 2013, 8 (7), 1381-1390. DOI: 10.1038/nprot.2013.081.

26. Bolisetty, S.; Arcari, M.; Adamcik, J.; Mezzenga, R., Hybrid Amyloid Membranes for Continuous Flow Catalysis. Langmuir 2015, 31 (51), 13867-13873. DOI:

10.1021/acs.langmuir.5b03205.

27. Li, C.; Adamcik, J.; Mezzenga, R., Biodegradable nanocomposites of amyloid fibrils and graphene with shape-memory and enzyme-sensing properties. Nat Nano 2012, 7 (7), 421-427.

28. Li, C.; Bolisetty, S.; Mezzenga, R., Hybrid Nanocomposites of Gold Single-Crystal Platelets and Amyloid Fibrils with Tunable Fluorescence, Conductivity, and Sensing Properties. Advanced Materials 2013, 25 (27), 3694-3700. DOI: 10.1002/adma.201300904.

29. Li, C.; Born, A.-K.; Schweizer, T.; Zenobi-Wong, M.; Cerruti, M.; Mezzenga, R., Amyloid-Hydroxyapatite Bone Biomimetic Composites. Advanced Materials 2014, 26 (20), 3207-3212. DOI: 10.1002/adma.201306198. 
30. Ling, S.; Li, C.; Adamcik, J.; Shao, Z.; Chen, X.; Mezzenga, R., Modulating Materials by Orthogonally Oriented $\beta$-Strands: Composites of Amyloid and Silk Fibroin Fibrils. Advanced Materials 2014, 26 (26), 4569-4574. DOI: 10.1002/adma.201400730.

31. Zakeri, B.; Fierer, J. O.; Celik, E.; Chittock, E. C.; Schwarz-Linek, U.; Moy, V. T.; Howarth, M., Peptide tag forming a rapid covalent bond to a protein, through engineering a bacterial adhesin. Proceedings of the National Academy of Sciences 2012, 109 (12), E690-E697. DOI: 10.1073/pnas.1115485109.

32. Nagai, T.; Ibata, K.; Park, E. S.; Kubota, M.; Mikoshiba, K.; Miyawaki, A., A variant of yellow fluorescent protein with fast and efficient maturation for cell-biological applications. Nat Biotech 2002, 20 (1), 87-90.

33. Hammer, N. D.; Schmidt, J. C.; Chapman, M. R., The curli nucleator protein, CsgB, contains an amyloidogenic domain that directs CsgA polymerization. Proceedings of the National Academy of Sciences of the United States of America 2007, 104 (30), 12494-12499. DOI: 10.1073/pnas.0703310104.

34. Wang, X.; Hammer, N. D.; Chapman, M. R., The Molecular Basis of Functional Bacterial Amyloid Polymerization and Nucleation. The Journal of Biological Chemistry 2008, 283 (31), 21530-21539. DOI: 10.1074/jbc.M800466200.

35. Andreev, K.; Bianchi, C.; Laursen, J. S.; Citterio, L.; Hein-Kristensen, L.; Gram, L.; Kuzmenko, I.; Olsen, C. A.; Gidalevitz, D., Guanidino groups greatly enhance the action of antimicrobial peptidomimetics against bacterial cytoplasmic membranes. Biochimica et Biophysica Acta (BBA) - Biomembranes 2014, 1838 (10), 2492-2502.

36. Gallo, P. M.; Rapsinski, G. J.; Wilson, R. P.; Oppong, G. O.; Sriram, U.; Goulian, M.; Buttaro, B.; Caricchio, R.; Gallucci, S.; Tükel, Ç., Amyloid-DNA composites of bacterial biofilms stimulate autoimmunity. Immunity 2015, 42 (6), 1171-1184. DOI: 10.1016/j.immuni.2015.06.002.

37. Rashid, F.; Sharma, S.; Bano, B., Comparison of Guanidine Hydrochloride (GdnHCl) and Urea Denaturation on Inactivation and Unfolding of Human Placental Cystatin (HPC). The Protein Journal 2005, 24 (5), 283-292. DOI: 10.1007/s10930-005-6749-5.

38. Camilloni, C.; Rocco, A. G.; Eberini, I.; Gianazza, E.; Broglia, R. A.; Tiana, G., Urea and Guanidinium Chloride Denature Protein L in Different Ways in Molecular Dynamics Simulations. Biophysical Journal 2008, 94 (12), 4654-4661. DOI: 10.1529/biophysj.107.125799. 39. Domachuk, P.; Perry, H.; Amsden, J. J.; Kaplan, D. L.; Omenetto, F. G., Bioactive "selfsensing” optical systems. Applied Physics Letters 2009, 95 (25), 253702.

40. White, A. P.; Gibson, D. L.; Collinson, S. K.; Banser, P. A.; Kay, W. W., Extracellular Polysaccharides Associated with Thin Aggregative Fimbriae of Salmonella enterica Serovar Enteritidis. Journal of Bacteriology 2003, 185 (18), 5398-5407. DOI: 10.1128/jb.185.18.53985407.2003.

41. Lim, J. Y.; Pinkner, J. S.; Cegelski, L., Community behavior and amyloid-associated phenotypes among a panel of uropathogenic E. coli. Biochemical and Biophysical Research Communications 2014, 443 (2), 345-350. 
42. Jackson, M.; Mantsch, H. H., The Use and Misuse of FTIR Spectroscopy in the Determination of Protein Structure. Critical Reviews in Biochemistry and Molecular Biology 1995, 30 (2), 95-120. DOI: 10.3109/10409239509085140.

43. Kong, J.; Yu, S., Fourier Transform Infrared Spectroscopic Analysis of Protein Secondary Structures. Acta Biochimica et Biophysica Sinica 2007, 39 (8), 549-559. DOI: 10.1111/j.1745-7270.2007.00320.x.

Table of content graphic

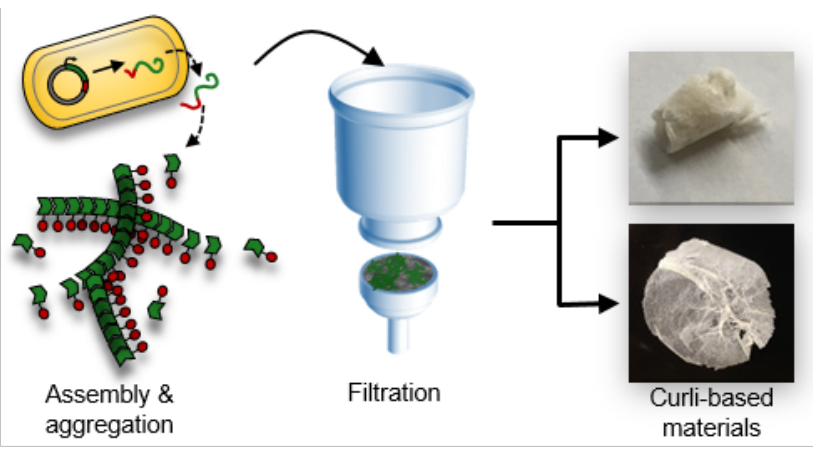

